\title{
Fungus Balls of the Bilateral Paranasal Sinuses
}

\author{
Dong Hoon Lee $\cdot$ Young Eun Joo $\cdot$ Sang Chul Lim
}

Received: 26 September 2011 / Accepted: 26 December 2011/Published online: 24 January 2012

(C) Association of Otolaryngologists of India 2012

\begin{abstract}
We describe the clinical and radiographic characteristics of fungus balls in the bilateral paranasal sinuses. The medical records of 8 of 245 patients with fungus balls of the bilateral paranasal sinuses between 2000 and 2010 were retrospectively reviewed. The incidence of bilateral paranasal sinus fungus balls was 3.3\%. Fungus balls were located in the maxillary sinuses bilaterally in 4 cases $(50 \%)$, followed by the maxillary sinus and contralateral sphenoid sinus in 3 cases $(37.5 \%)$, and the sphenoid sinuses bilaterally in 1 case $(12.5 \%)$. There were no predisposing anatomic variations for the occurrence of bilateral paranasal fungus balls. Although the presenting symptoms and signs were non-specific, CT findings were helpful in the diagnosis of bilateral fungus balls. Endonasal removal by an endoscopic approach was performed in all patients. No peri-operative complications or recurrences were noted. Fungus balls in the bilateral paranasal sinuses are most frequently found in the maxillary sinuses bilaterally. Because symptoms of bilateral paranasal fungus balls and findings on nasal endoscopic examination are
\end{abstract}

D. H. Lee - S. C. Lim

Department of Otolaryngology-Head and Neck Surgery, Chonnam National University Medical School and Chonnam National University Hwasun Hospital, Hwasun, South Korea e-mail: leen31@hanmail.net

\section{S. C. Lim $(\bowtie)$}

Department of Otolaryngology-Head and Neck Surgery, Chonnam National University Medical School and Chonnam National University Hwasun Hospital, 160 Ilsimri, Hwasun, Jeollanamdo 519-809, South Korea

e-mail: limsc@chonnam.ac.kr

Y. E. Joo

Research Institute of Medical Sciences, Chonnam National University Medical School, Gwangju, South Korea frequently non-specific, a high index of suspicion is needed and imaging studies, such as CT, are essential to establish the correct pre-operative diagnosis.

Keywords Fungus disease - Sinusitis - Bilateral · Paranasal sinuses $\cdot$ Endoscopic surgical procedure

\section{Introduction}

The fungus ball of the paranasal sinuses is a non-invasive form of fungal sinusitis occurring in immunocompetent hosts [1]. Fungus balls (FBs) are characterized by a mass of inspissated fungal debris and mucus progressively growing into the sinus cavity without invasion of the underlying mucosa [2]. FBs are usually found in only one sinus; FBs are most frequently found in the maxillary sinus [3]. Multiple sinus involvement by FBs has been reported to occur in $6.4 \%$ of cases in the largest review of this disease entity [3]. Although FBs can be involved in multiple sinuses, these sinuses are mostly contiguous. Bilateral paranasal sinus involvement is rare [3-5], which has not been described in detail.

In this study, we report the largest case series with respect to clinical and radiographic characteristics and treatment outcomes of FBs of the bilateral paranasal sinuses.

\section{Patients and Methods}

After obtaining Institutional Review Board (IRB) approval, we identified 245 patients with paranasal sinus FBs who were treated at Chonnam National University Hostpital and Hwasun Hospital between January 2000 and October 2010. Among the 245 patients, 8 had FBs of the bilateral 
paranasal sinuses and the medical records were reviewed retrospectively. The data collected included medical and surgical histories, presenting symptoms, physical examination findings, radiographic findings, complications, and surgical outcome.

Surgery was performed via an endoscopic approach. When the FBs were localized in the maxillary sinus, a uncinectomy and maxillary antrostomy were performed, and all of the fungal debris was removed. A sphenoidotomy was performed via the transnasal or transethmoidal approach. The fungal materials were removed and sent for pathologic examination, and fungus cultures were performed if necessary. The nasal cavities were packed with Merocel (Medtronic, Jacksonville, FL, USA), which was removed on post-operative day 1 .

\section{Results}

Eight patients ( 4 males and 4 females) were included in the study and 16 isolated sinuses were affected. The age of the patients ranged between 42 and 69 years, with a mean of 59.3 years. The underlying diseases included diabetes, hypertension, and lung cancer in 3 patients. The incidence of bilateral paranasal sinus FBs was 3.3\%.

Patients presented with a chief complaint of purulent rhinorrhea $(75 \%)$, nasal obstruction $(38 \%)$, or headaches (25\%). Endoscopic findings of the middle or superior meatus revealed polyps, discharge, or swelling in 9 sides; abnormal findings were not shown in 7 sides. All patients underwent pre-operative computed tomography (CT) of the paranasal sinuses (Fig. 1). Bilateral maxillary sinuses were the most common pattern of involvement (4 of 8 patients [50\%]), followed by the maxillary sinus and contralateral sphenoid sinus ( 3 of 8 patients [37.5\%]), and bilateral sphenoid sinuses ( 1 of 8 cases [12.5\%]). The CT findings of maxillary FBs included microcalcifications (8 of 11 involved sinuses [73\%]), diffuse thinning of the medial wall ( 1 of 11 involved sinuses [9\%]), and sinus wall sclerosis (7 of 11 involved sinuses [64\%]). The CT findings of sphenoid FBs included sinus wall sclerosis (5 of 5 involved sinuses [100\%]) focal erosions (3 of 5 involved sinuses [60\%]), and calcifications (3 of 5 involved sinuses [60\%]). The anatomic variations associated with FBs were examined. Septal deviation and concha bullosa were noted in each 3 of 8 patients, but these variations were not related to the development of maxillary FBs. Despite no evidence of endodontic treatment, all five dentulous patients had FBs, which may suggest low probability of the causal relationship between endodontic treatment and FB.

All patients underwent removal of FBs through an endoscopic approach. A middle meatal antrostomy and removal of the hyphal mass was performed in maxillary

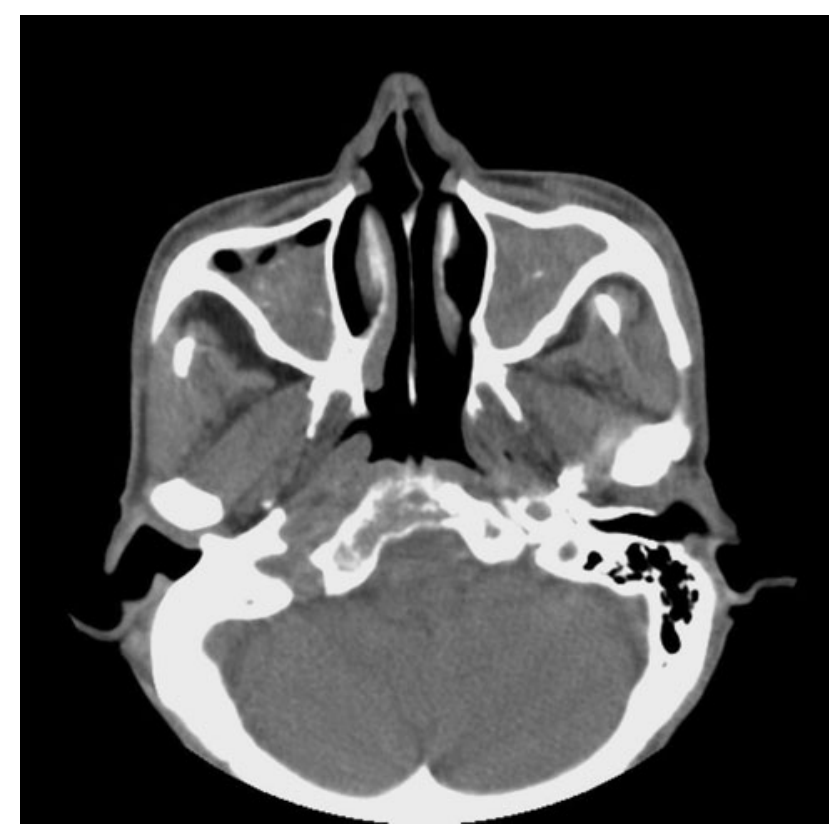

Fig. 1 A CT scan shows a typical appearance of fungus ball in both maxillary sinuses (microcalcifications and sinus wall sclerosis)

FBs and a transnasal approach was performed in all patients with sphenoid FBs. Histopathologic examination of the specimens demonstrated Aspergillus species in all cases. Fungal cultures were performed in two cases, but there was no fungal growth. No peri-operative complications were noted, and there were no recurrences during the follow-up, which ranged from 8 to 61 months (mean, 30 months). The involved sinuses and clinical summary are shown in Table 1.

\section{Discussion}

Paranasal sinus fungal infection is an uncommon disease; however, the incidence may be increasing for a number of reasons, including better detection through endoscopic and radiologic evaluation and longer life expectancies [6]. FBs are an extramucosal fungal proliferation usually occurring in immunocompetent individuals as a maxillary sinus involvement caused by Aspergillus species [4]. Because FBs are usually unilateral paranasal sinus lesions, bilateral involvement is rare. In the largest series involving FBs of the paranasal sinuses, 2 of 109 patients $(1.8 \%)$ had bilateral maxillary sinus involvement [3]. Nicholai et al. [2] and Ferreiro et al. [5] reported 2 of 160 patients (1.25\%) and 1 of 29 patients $(3.4 \%)$ with bilateral paranasal sinuses involvement, respectively. In our study, 8 of 245 patients (3.3\%) had bilateral paranasal sinuses involvement.

The pathogenesis of FBs is unknown. A possible contributing factor of FBs may be ostio-meatal complex 
Table 1 Summary of fungus balls of the bilateral paranasal sinsuses
$F B$ fungus ball, $D M$ diabetes, $H T N$ hypertension, $M$ maxillary sinus, $S$ sphenoid sinus, $N E D$ no evidence of disease

\begin{tabular}{llllllll}
\hline Case & $\begin{array}{l}\text { Age/ } \\
\text { gender }\end{array}$ & $\begin{array}{l}\text { Underlying } \\
\text { diseases }\end{array}$ & $\begin{array}{l}\text { Involved } \\
\text { site }(\mathrm{R} / \mathrm{L})\end{array}$ & $\begin{array}{l}\text { Septal } \\
\text { deviation }\end{array}$ & $\begin{array}{l}\text { Concha } \\
\text { bullosa }\end{array}$ & $\begin{array}{l}\text { Endodontic } \\
\text { treatment }\end{array}$ & $\begin{array}{l}\text { Follow-up } \\
\text { (months) }\end{array}$ \\
\hline 1 & $69 / \mathrm{M}$ & DM, HTN & M/S & Left side & None & Edentulous & NED (53) \\
2 & $63 / \mathrm{F}$ & DM, HTN & S/S & None & None & None & NED (61) \\
3 & $50 / \mathrm{M}$ & None & S/M & None & Both & None & NED (38) \\
4 & $64 / \mathrm{F}$ & None & S/M & None & None & Edentulous & NED (37) \\
5 & $62 / \mathrm{M}$ & None & M/M & Left side & None & None & NED (12) \\
6 & $57 / F$ & None & M/M & None & None & None & NED (10) \\
7 & $42 / F$ & None & M/M & Left side & Both & None & NED (24) \\
8 & $67 / M$ & Lung ca & M/M & None & None & None & NED (8) \\
\hline
\end{tabular}

in $81 \%$ of cases of non-fungal sinusitis. Punctuate microcalcifications occurred were most frequently in maxillary FBs, and in contrast, smooth marginated calcifications occurred more commonly in maxillary non-fungal sinusitis [10]. Sinus wall sclerosis was one of the common findings and the presence of both calcifications and sinus wall sclerosis was highly suggestive of bilateral maxillary FBs. The CT findings of sphenoid FBs was somewhat different from of the CT findings of maxillary FBs; specifically, sinus wall sclerosis was the most common finding on CT of maxillary FBs. In contrast to maxillary FBs, focal bony erosions appeared to be more common in sphenoid FBs, which may be attributable to expansion of FBs within the relatively small sphenoid sinus cavity.

Histopathologic examination, by assessing the presence of fungal hyphae in the specimens and excluding mucosal invasion, is essential for the diagnosis of FBs [2]. FBs are usually caused by Aspergillus species [4]. In our study, histopathologic examination of FBs with bilateral paranasal sinuses demonstrated Aspergillus species in all cases. Fungal culture is useful to identify fungal species, but failure of the fungus to grow on fungal culture is common. The treatment of choice for FBs of the paranasal sinuses is complete removal of FBs from the involved sinus to reestablish proper ventilation and drainage [11]. The endoscopic approach has been used as the primary treatment for FBs of the paranasal sinuses and has the advantage for easy access to the affected sinus, perfect visualization into the sinus, and low morbidity [1]. Our patients underwent endoscopic treatment and had excellent treatment outcomes without recurrence.

\section{Conclusion}

FBs of the bilateral paranasal sinuses are a rare presenting form of paranasal FBs. Because of non-specific symptoms and frequent negative endoscopic findings, a high index of suspicion is required in the diagnosis of bilateral paranasal FBs. To establish the correct pre-operative diagnosis, imaging studies, such as a CT scan, are essential. 


\section{Key Messages}

- The incidence of bilateral paranasal sinus fungus balls was $3.3 \%$.

- Fungus balls in the bilateral paranasal sinuses are most frequently found in the maxillary sinuses bilaterally.

- Because symptoms of bilateral paranasal fungus balls and findings on nasal endoscopic examination are frequently non-specific, a high index of suspicion is needed and imaging studies, such as CT, are essential to establish the correct pre-operative diagnosis.

\section{References}

1. Dufour X, Kauffmann-Lacroix C, Ferrie JC, Goujon JM, Rodier MH, Karkas A et al (2005) Paranasal sinus fungus ball and surgery: a review of 175 cases. Rhinology 43:34-39

2. Nicolai P, Lombardi D, Tomenzoli D, Villaret AB, Piccioni M, Mensi $M$ et al (2009) Fungus ball of the paranasal sinuses: experience in 160 patients treated with endoscopic surgery. Laryngoscope 119:2275-2279

3. Klossek JM, Serrano E, Péloquin L, Percodani J, Fontanel JP, Pessey JJ et al (1997) Functional endoscopic sinus surgery and 109 mycetomas of paranasal sinuses. Laryngoscope 107:112-117
4. deShazo RD, O’Brien M, Chapin K, Soto-Aguilar M, Swain R, Lyons $M$ et al (1997) Criteria for the diagnosis of sinus mycetoma. J Allergy Clin Immunol 99:475-485

5. Ferreiro JA, Carlson BA, Cody DT 3rd (1997) Paranasal sinus fungus balls. Head Neck 19:481-486

6. Robey AB, O'Brien EK, Richardson BE, Baker JJ, Poage DP, Leopold DA (2009) The changing face of paranasal sinus fungus balls. Ann Otol Rhinol Laryngol 118:500-505

7. Tsai TL, Guo YC, Ho CY, Lin CZ (2006) The role of ostiomeatal complex obstruction in maxillary fungus ball. Otolaryngol Head Neck Surg 134:494-498

8. Legent F, Billet J, Beauvillain C, Bonnet J, Miegeville M (1989) The role of dental canal fillings in the development of aspergillus sinusitis. A report of 85 cases. Arch Otorhinolaryngol 246:318320

9. Roithmann R, Shankar L, Hawke M, Chapnik J, Kassel E, Noyek A (1995) Diagnostic imaging of fungal sinusitis: eleven new cases and literature review. Rhinology 33:104-110

10. Yoon JH, Na DG, Byun HS, Koh YH, Chung SK, Dong HJ (1999) Calcification in chronic maxillary sinusitis: comparison of CT findings with histopathologic results. AJNR Am J Neuroradiol 20:571-574

11. Dufour X, Kauffmann-Lacroix C, Ferrie JC, Goujon JM, Rodier MH, Klossek JM (2006) Paranasal sinus fungus ball: epidemiology, clinical features and diagnosis. A retrospective analysis of 173 cases from a single medical center in France, 1989-2002. Med Mycol 44:61-67 